\title{
Geriatric Cerebrovascular Neurosurgery: Institutional Experience from Khoula Hospital
}

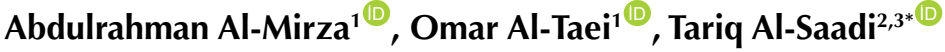 \\ ${ }^{1}$ Sultan Qaboos University College of Medicine, Muscat, Sultanate of Oman \\ ${ }^{2}$ Department of Neurology \& Neurosurgery - Montreal Neurological Institute, Faculty of Medicine, \\ McGill University, QC, Canada \\ ${ }^{3}$ Department of Neurosurgery, Khoula Hospital, Muscat, Sultanate of Oman
}

\begin{abstract}
Background: The aging of the healthy population without severe morbidity exposes them to cerebrovascular diseases and demand effective management. This study aimed to study the prevalence of geriatric cerebrovascular neurosurgical conditions in the Neurosurgical Department at Khoula Hospital, Muscat, Sultanate of Oman.

Methods: A retrospective chart review of was done on geriatric cases admitted to the Neurosurgery Department in Khoula Hospital as an example of a neurosurgical center in a high-income developing country from January 2016 to 31st December 2019. Patients'

demographics, risk factors, diagnosis, Glasgow Coma Scale (GCS) on arrival, medications used, and length of stay were recorded.

Results: 109 patients aged over 65 years were recruited in our retrospective review with a mean age of 74.12 years. Male-to-female ratio was (1.2:1). Intracerebral hemorrhage (ICH) was the most common vascular diagnosis $(39.0 \%)$ followed by subarachnoid hemorrhage (SAH) $(22.9 \%)$. Most patients $(41.9 \%)$ had a GCS score of less than 8 . About one-fifth of the patients received antiplatelet and anticoagulant medications. Most of the patients underwent surgical intervention $(61.9 \%)$. $59 \%$ of the patients stayed in the hospital for less than 15 days. There were significant associations between the length of stay, treatment types (surgical vs. conservative), and age $(P<0.05)$.

Conclusion: Cerebrovascular pathologies are a growing cause of mortality and morbidity worldwide including developing countries because of the increasing number of elderly people. Antiplatelet medication and anticoagulants should be used with caution in the elderly.

Keywords: Cerebrovascular surgery; Geriatric population; Haemorrhage.
\end{abstract}

*Correspondence to Tariq Al-Saadi,

MD, Department of Neurology \& Neurosurgery - Montreal Neurological Institute, Faculty of Medicine, McGill University, QC, Canada and Department of Neurosurgery, Khoula Hospital, Muscat, Sultanate of Oman Email: t.dhiyab@hotmail.com

Published online June 30, 2021

Citation: Al-Mirza A, Al-Taei O, Al-Saadi T. Geriatric cerebrovascular neurosurgery: institutional experience from khoula hospital. Clin Neurosci J. 2021;8(3):129-134. doi:10.34172/icnj.2021.27.

\section{Introduction}

The aging of the healthy population without severe morbidity exposes them to several vascular accidents such as subarachnoid hemorrhage (SAH), intracerebral hemorrhage (ICH), cerebral aneurysm because of fragility of the blood vessels, and other vascular pathologies associated with age that demand effective management. ${ }^{1}$ The yearly incidence of SAH in the elderly population ranges from 1.3 to $1.6 \% .^{1}$ The average case fatality for $\mathrm{SAH}$ is around $50 \% .^{1} \mathrm{SAH}$ is defined as direct extravasation of blood into the subarachnoid space caused by the rupture of lesional vessels located at the brain or surface. ${ }^{2}$ Age has an independent negative influence on the outcome. ${ }^{3}$ The condition is common in the elderly compared to the younger population due to parenchymal atrophy, which allows a large quantity of blood to collect after aneurysm rupture. $^{3}$ This accounts for $5 \%$ of all acute strokes. ${ }^{3}$ Mortality rates as high as $45 \%$ have also been reported. ${ }^{3}$ Multiple risk factors are involved in $\mathrm{SAH}$, including diabetes, myocardial disease, arterial hypertension, cerebrovascular and pulmonary disease, and smoking. ${ }^{3}$ Aneurysmal SAH, is well-known to rise with age, especially in women. The number of elderly patients with aneurysmal SAH and the total prevalence are on the rise. ${ }^{4}$ Multiple studies have indicated that poor outcomes increase in patients over the age of $75 .{ }^{4}$ Moreover, aneurysmal SAH has high mortality and severe disability rates than other types of SAH types. ${ }^{2}$ The headache associated with aneurysmal SAH is usually so severe that codeine or even a synthetic opiate might be needed. ${ }^{3}$ It is the most common cause of sudden death. ${ }^{2}$ Moreover, the size of an aneurysm appears to be a prognostic factor in aneurysmal SAH. ${ }^{2} \mathrm{ICH}$ is a term indicating numerous different conditions, including haemorrhagic stroke, subdural hematoma, and epidural hematoma. Almost $20 \%$ of all strokes are related to $\mathrm{ICH} .{ }^{5} \mathrm{ICH}$ is one of the utmost serious complications of oral anticoagulants and anti-platelets, and mortality rates are over $50 \% .^{5}$ 
Intraventricular hemorrhage is a rare subtype of $\mathrm{ICH}$, in which bleeding is confined to the ventricular system without parenchymal or subarachnoid involvement. ${ }^{6}$ It is estimated that intraventricular hemorrhage comprises $3.1 \%-9 \%$ of all $\mathrm{ICH}{ }^{6}$

We aimed to analyse the prevalence and outcome of cerebrovascular neurosurgical conditions in the geriatric population in a tertiary hospital in the Sultanate of Oman. The health care services in Oman are considered as having one of the best health care systems in the world according to World Health Organization reports. ${ }^{7,8}$ The Department of Neurosurgery in our hospital is the main neurosurgical center in the country with an average annual admission of 1600 patients. ${ }^{9,10}$ In this study, we chose a cut of 65 years and older according to the local definition, taking into account the increase in life span throughout the last decades as well as the improvement in the quality of life.

\section{Patients and Methods}

This was a retrospective study conducted at Khoula Hospital located in Muscat, Sultanate of Oman. Medical records of 669 patients over the age of 65 admitted to the neurosurgical ward were reviewed. Among them, 109 were diagnosed with vascular non-traumatic conditions. Patients admitted from the period of January 2016 to $31^{\text {st }}$ December 2019 were included. The study includes both Omani and non-Omani patients. The exclusion criteria were as follows: non-elderly patient (below 65 years), non-neurosurgical conditions and neurosurgical conditions other than vascular diseases, patients with traumatic cerebrovascular conditions, outside the study period (from January 1, 2016 to December 31, 2019), and patients with missing or incomplete data.

Data were obtained from the health information system including patient demographics (age, sex), Glasgow Coma Score (GCS) on arrival, diagnosis, length of hospital stay (LOS), and treatment proposed. Then the information is classified into continuous and categorized variables and analyzed accordingly.

The research database was analyzed and processed using SPSS software (version 23). The categorized variables were cross-tabulated using frequency tables and pie charts or bar charts. A chi-square test was used to obtain the significance of the association between categorized variables, using a $P \leq 0.05$ as the cut-off for significance. The numerical variables were summarized by their medians, means, and ranges, and the categorical variables were described by their counts and relative frequencies.

\section{Results}

Table 1 shows the demographic characteristics of the included cases in the present study. We have a total of 669 patients admitted to the neurosurgical department at Khoula Hospital in Muscat the capital city of Sultanate of Oman in four years' period (from 2016 to 2019). Of those 669 patients, 105 patients were admitted because of vascular non-traumatic lesions, which will be the main focus of our study. $65.7 \%$ of the study cohort were more than 75-year-old. The male to female ratio was (1.2:1). $\mathrm{ICH}$ was the most common vascular diagnosis (39.0\%)

Table 1. Demographic Characteristics of the Patients

\begin{tabular}{|c|c|}
\hline Category & $\begin{array}{c}\text { Number of patients } \\
(\%)\end{array}$ \\
\hline \multicolumn{2}{|l|}{ Number of patients admitted each year } \\
\hline 2019 & $202(30.0 \%)$ \\
\hline 2018 & $172(25.7 \%)$ \\
\hline 2017 & $154(23 \%)$ \\
\hline 2016 & $141(21.3 \%)$ \\
\hline $\begin{array}{l}\text { Total number of admitted neurosurgical } \\
\text { cases (2016-2019) }\end{array}$ & 669 \\
\hline Total number of vascular cases & 105 \\
\hline \multicolumn{2}{|l|}{ Age } \\
\hline$\geq 75$ & $36(34.3 \%)$ \\
\hline$<75$ & $69(65.7 \%)$ \\
\hline \multicolumn{2}{|l|}{ Gender } \\
\hline Female & $48(45.7 \%)$ \\
\hline Male & $57(54.3 \%)$ \\
\hline \multicolumn{2}{|l|}{ Types of vascular diagnosis } \\
\hline $\mathrm{ICH}$ & $41(39.0 \%)$ \\
\hline $\mathrm{SAH}$ & $24(22.9 \%)$ \\
\hline Aneurysms & $15(14.3 \%)$ \\
\hline Ischemic lesions & $14(13.3 \%)$ \\
\hline Multi-compartmental haemorrhage & $8(7.6 \%)$ \\
\hline Arteriovenous malformation & $3(2.9 \%)$ \\
\hline \multicolumn{2}{|l|}{ GCS on arrival } \\
\hline $15-14$ & $42(39.9 \%)$ \\
\hline $13-12$ & $9(8.5 \%)$ \\
\hline $11-9$ & $10(9.5 \%)$ \\
\hline$\leq 8$ & $44(41.9 \%)$ \\
\hline \multicolumn{2}{|l|}{ Use of antiplatelet drugs } \\
\hline Yes & $19(18.1 \%)$ \\
\hline No & $86(81.9 \%)$ \\
\hline \multicolumn{2}{|l|}{ Use of anticoagulant drugs } \\
\hline Yes & $23(21.9 \%)$ \\
\hline No & $82(78.1)$ \\
\hline \multicolumn{2}{|l|}{ Type of interventions } \\
\hline Surgical & $65(61.9 \%)$ \\
\hline Conservative & $40(38.1 \%)$ \\
\hline \multicolumn{2}{|l|}{ LOS } \\
\hline$\leq 15$ days & $65(61.9 \%)$ \\
\hline$>15$ days & $40(38.1 \%)$ \\
\hline
\end{tabular}


followed by SAH (22.9\%). Most patients had a GCS score of less than 8 (41.9\%). $18.1 \%$ of the patients received antiplatelet medications, most commonly aspirin. $21.9 \%$ of the patients received anticoagulant medications, most commonly enoxaparin. Most patients (61.9\%) underwent surgical intervention. 59\% of the patients stayed in the hospital for less than 15 days.

Figure 1 represents the total number of admitted patients with vascular lesions each year. As shown, vascular cases continuously increased among the study years with the highest number of cases in 2019 ( $n=33,31.4 \%)$

The association between the age of the patients with vascular pathologies and other variables (use of antiplatelet, use of anticoagulant and GCS) is shown in Table 2. The table demonstrates that there was no significant difference between the age of patients above and below 75 years and the use of antiplatelet and anticoagulant agents $(P=0.795, P=0.660$, respectively), yet, the use of both groups of medications was higher in the older age group (more than 75 years). Also, it shows that there was no significant association between age above and below 75 years and GCS score (above and below 8); So, both age groups presented to the hospital with a similar level of consciousness $(P=0.385)$.

Table 3 demonstrates the association between the LOS of the patients and other variables (age and type of intervention). There was a significant relationship between the age of the patients (more and less than 75 years) and LOS (15 days as a cut-off value) $(P=0.047)$. Also, there was a significant difference between LOS and the type of intervention (surgical vs. conservative), in which the patients with a conservative type of management found to have a shorter LOS in the hospital compared with the patients who were underwent surgical interventions $(P<0.005)$.

Figure 2 represents the average age of the patients among the different types of vascular cases, in which patients with ICH were found to have the highest mean age (75.3

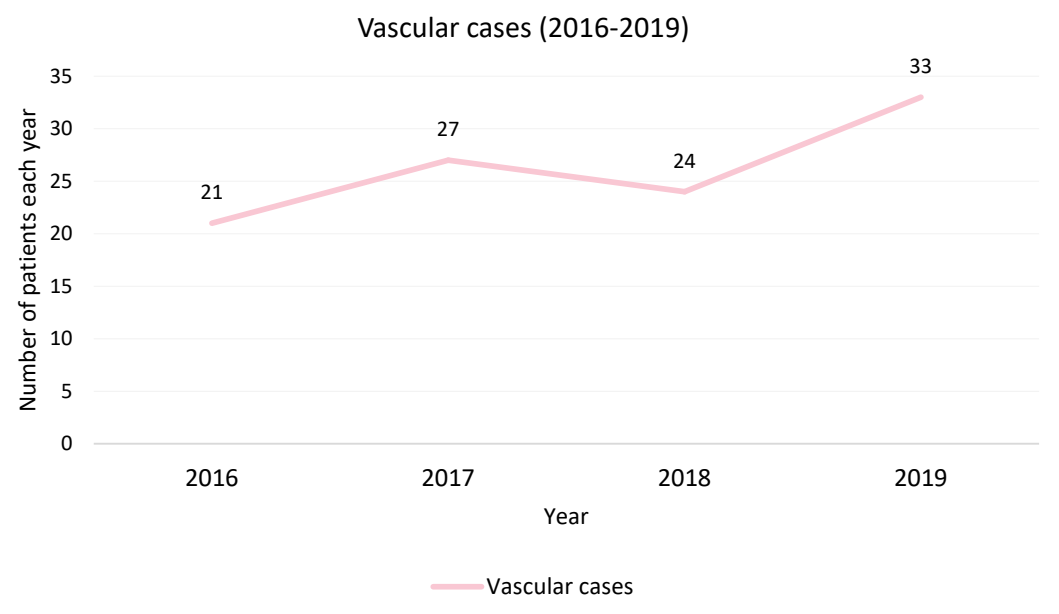

Figure 1. Total Number of Admitted Patients With Vascular Cases Each Year.

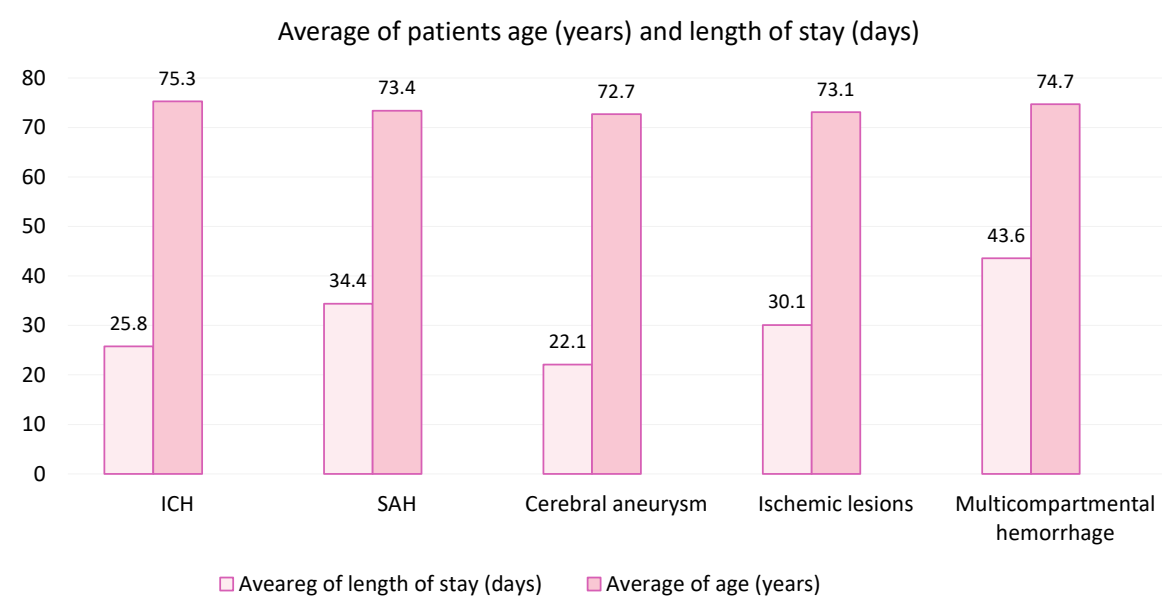

Figure 2. Average Age (years) of the Patients and Length of Stay (Days) in Different Types of Vascular Cases. Abbreviations: ICH, intracerebral hemorrhage; SAH, subarachnoid hemorrhage; LOS, length of stay. 
Table 2. The Association Between the Age of the Patients and Other Variables (Use of Antiplatelet, Use of Anticoagulants, and GCS)

\begin{tabular}{|c|c|c|c|c|c|c|c|}
\hline & & \multicolumn{2}{|c|}{ Use of antiplatelet } & \multicolumn{2}{|c|}{ Use of anticoagulant } & \multicolumn{2}{|c|}{ GCS } \\
\hline & & Yes & No & Yes & No & GCS $<8$ & GCS $>8$ \\
\hline \multirow{2}{*}{ Age } & $<75$ & 7 & 29 & 7 & 29 & 13 & 23 \\
\hline & $\geq 75$ & 12 & 57 & 16 & 53 & 31 & 38 \\
\hline$P$ value & & \multicolumn{2}{|c|}{0.795} & \multicolumn{2}{|c|}{0.660} & \multicolumn{2}{|c|}{0.385} \\
\hline
\end{tabular}

Table 3. The Association Between the LOS of the Patients and Other Variables (Age and Type of Intervention)

\begin{tabular}{lcccc}
\hline \multirow{2}{*}{ LOS } & \multicolumn{2}{c}{ Age $(\mathbf{y})$} & \multicolumn{2}{c}{ Type of intervention } \\
\cline { 2 - 5 } & $<75$ & $\geq 75$ & Surgical & Conservative \\
\hline Less than 15 days & 26 & 36 & 31 & 31 \\
More than 15 days & 36 & 33 & 34 & 9 \\
$P$ value & \multicolumn{2}{c}{0.047} & & 0.003 \\
\hline
\end{tabular}

Abbreviation: LOS, length of stay

years) followed by multi-compartmental hemorrhage and SAH, respectively (74.7 years, 73.4 years). Moreover, patients with multi-compartmental hemorrhage had the longest LOS in the hospital (mean: 43.6 days) followed by SAH (mean: 34.4 days).

\section{Discussion}

The main focus of this study was to investigate the prevalence, etiological factors, and consequences related to the different types of vascular pathologies in the geriatric population in the Sultanate of Oman. In the present study, almost two-thirds of the patients were more than 75 -year-old. This is due to the increase in life expectancy all over the world including the developing countries due to advancement in the healthcare sector and method of delivery of treatment. Compared to another study done by Guo et al.that demonstrated an average of 67.9 years for the studied cohort. ${ }^{6}$ In our study, male to female ratio was $(1.2: 1)$ which goes in the same line to the Omani count population that states that the male to female ratio in Oman is 180.8 males per 100 females, ${ }^{11}$ in comparison to other studies in which the population is mostly females. ${ }^{6}$ The Omani populations' pyramid is shown in Figure 3 which shows that majority of the population is young age group, added to that the larger number of males compared to females. Comparative studies between the elderly versus younger age have indicated that women were predominant above the age of 65 years, 70 years, or

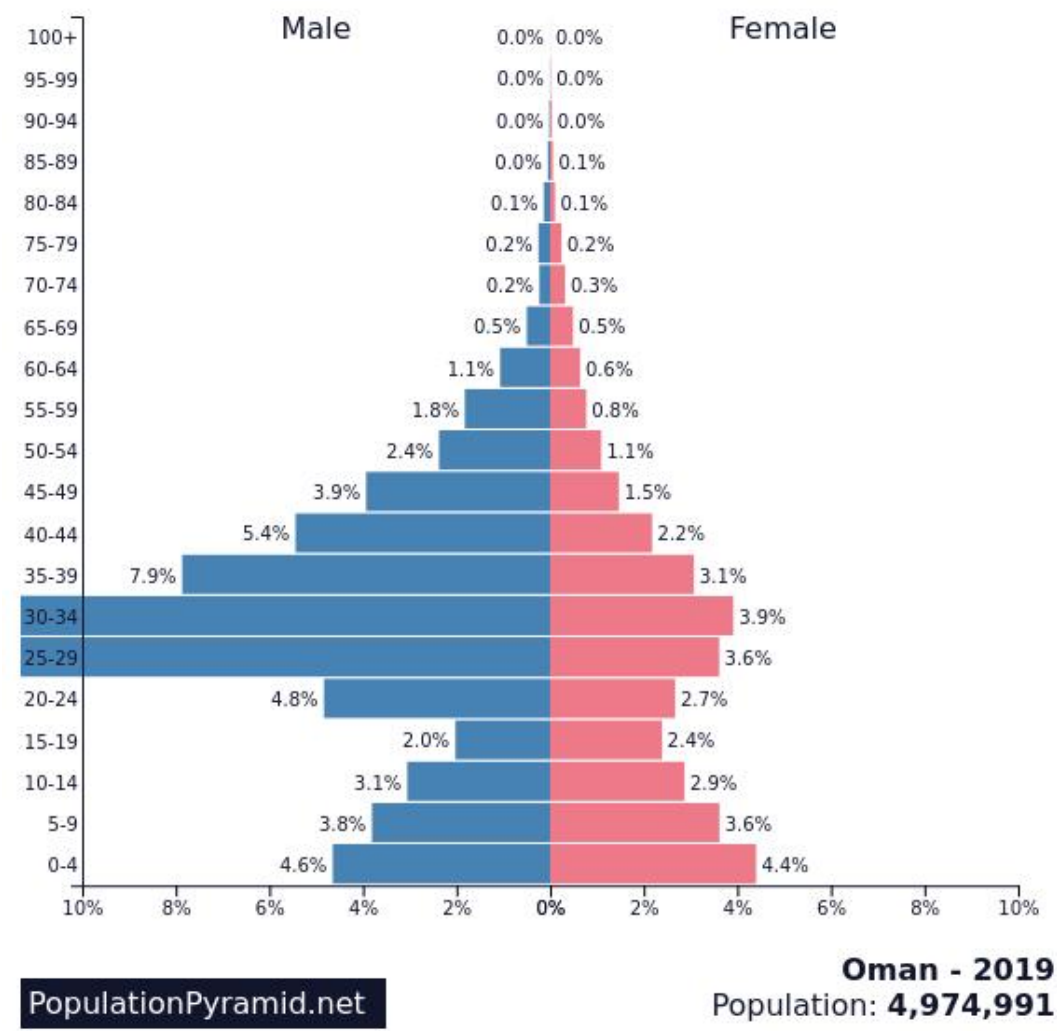

Figure 3. The Omani Populations Pyramid. 
with increasing age..$^{12,13}$

In the present study, ICH was the most common vascular diagnosis followed by $\mathrm{SAH}$. ICH is a growing cause of mortality and morbidity worldwide, because of the increasing number of elderly people. ${ }^{14}$ It is one of the important associated pathologies with strokes, as stated by Stein et al that approximately $20 \%$ of all strokes are due to ICH. ${ }^{12}$ However, it is also important to remember two other, less common vascular entities: subdural and epidural hematoma, which are both frequently associated with head trauma, especially in the elderly, yet, those two entities were not in the scope of this paper. Thus, they will be discussed in a separate paper that describes traumatic brain injury in a specialized way.

The GCS is considered to an important factor in the prognosis of the patients, as elderly patients with low GCS and coagulopathy had poor prognoses. ${ }^{12,14}$ Many patients had a GCS score of less than 8. In contrast with another study done by Cho and colleagues demonstrated a higher GCS score among both patients with a surgical and conservative type of management. ${ }^{14} \mathrm{~A}$ significant feature of elderly patients is often pre-existing health problems such as hypertension, diabetes mellitus, atherosclerosis, coronary heart disease, renal dysfunction, and other systemic diseases which are likely to be further exacerbated causing a deterioration in the patient's consciousness level.

About one-fifth of the patients received antiplatelet medications, most commonly aspirin. In the same line, a study done by Virta et al showed that $18.8 \%$ of the patients used antiplatelet agents. It is important to highlight the increased risk of vascular accidents among patients on antiplatelet therapy as its related to the co-existing medical conditions (such as cardiac and vascular diseases) that was found among $38.4 \%$ of patients. Therefore, antiplatelet therapy important factor in the overall clinical outcome. ${ }^{4,12}$

In the current study, almost one-fifth of the patients received anticoagulant medications, mostly enoxaparin. Prior research on the risks of anticoagulation in patients who experience head injury have reported mixed results. Though some studies suggest that the incidence of ICH is more common and outcomes were worse when they occur in patients who were on anticoagulation therapy. ${ }^{5}$

Most of the patients underwent surgical intervention in our review, in contrast to a previous study that showed that $61.8 \%$ of patients received conservative treatment and $38.2 \%$ of patients underwent at least one kind of surgical intervention. Active surgical treatment for some types of vascular entities such as aneurysms improves the prognoses of elderly patients compared with those who adopt a palliative treatment. ${ }^{1,4}$

In our study, $59 \%$ of the patients stayed in the hospital for less than 15 days, compared with a previous study showing an average of 20.1. ${ }^{5}$ Many factors affect recovery time such as the pre-existing comorbidities, surgical complications, and patients' age. ${ }^{5}$

We found no significant association between the age of the patients and the use of antiplatelet and anticoagulant agents. One of the most common clinical dilemmas in the practice of geriatrics is patients at high risk for falls who also have indications for anticoagulation. Clinicians often discontinue anticoagulants in older people who fall, especially among those who fall recurrently, out of concern for ICH and severe hematomas. We also found no significant association between age and the GCS scale, so, both age groups presented to the hospital with a similar level of consciousness, which depends upon many factors such as the type of vascular pathological entity, the duration of injury, and other factors. ${ }^{12}$

Patients with a conservative type of management found to have significantly shorter LOS in the hospital compared with those who underwent surgical intervention. Correspondingly, another study found in subgroup analysis that those who received the surgical intervention had better early and late functional outcomes which affected the overall physical improvement as well as LOS period at the hospital. ${ }^{14}$

Patients with ICH had the highest mean age. A previous study documented an average of 72.6 years for patients with ICH. Moreover, patients with multi-compartmental hemorrhage had the longest LOS in the hospital. This might be related to the generalized significant impact of the brain parenchyma and consequently the functional outcome among those patients. ${ }^{12}$

There were some limitations of this study. It was a single-centered retrospective study during four years. Thus, several limiting factors were found, such as the development in modern medical technology which provides better care for patients, and the availability of diagnostic facilities such as imaging facilities. The followup of the patients in the time after the study period was not assessed. Further studies needed to be conducted focusing on the impact of cerebrovascular diseases on the geriatric age group and the preventive measures that may reduce the incidence of those diseases and improve the overall clinical outcome.

\section{Conclusion}

Cerebrovascular pathologies are growing causes of mortality and morbidity worldwide, because of the increasing number of elderly people. In the present study, ICH was the most common vascular diagnosis. Most of the patients underwent surgical intervention. Special care must be taken when dealing with geriatric neurosurgical patients in general, and special attention should be paid to vascular cases because of higher rates of mortality and morbidity. 


\section{Conflict of Interest Disclosures}

No conflict of interest.

\section{Ethical Statement}

Ethical approval and consent to participate: No individual consent was required. The study was reviewed and approved by the Research Ethical Committee at Khoula Hospital/Ministry of health (PRO122020072).

\section{References}

1. Derrey S, Curey S, Hannequin P, Castel H, Langlois $\mathrm{O}$, Tollard E, et al. Elderly patients with aneurysmal subarachnoid hemorrhage: coils but also clips. Neurochirurgie. 2012;58(2-3):140-5. doi: 10.1016/j. neuchi.2012.02.014.

2. Zheng K, Zhao B, Tan XX, Li ZQ, Xiong Y, Zhong M, et al. Comparison of aggressive surgical treatment and palliative treatment in elderly patients with poor-grade intracranial aneurysmal subarachnoid hemorrhage. Biomed Res Int. 2018;2018:5818937. doi: 10.1155/2018/5818937.

3. Sedat J, Dib M, Rasendrarijao D, Fontaine D, Lonjon M, Paquis P. Ruptured intracranial aneurysms in the elderly: epidemiology, diagnosis, and management. Neurocrit Care. 2005;2(2):119-23. doi: 10.1385/ncc:2:2:119.

4. Ohkuma H, Shimamura N, Naraoka M, Katagai T. Aneurysmal subarachnoid hemorrhage in the elderly over age 75: a systematic review. Neurol Med Chir (Tokyo). 2017;57(11):575-83. doi: 10.2176/nmc.ra.2017-0057.

5. Angelozzi A, Renda G, Mercuri M, De Caterina R. The Risk of Intracranial Hemorrhage with Anticoagulation in the Elderly - Estimates of Prevalence and Therapeutic Strategies. American College of Cardiology; 2015

6. Guo R, Chen R, Yu Z, Tian R, Ren Y, You C, et al. Clinical features and prognosis of primary intraventricular hemorrhage in elderly: single-center experience. World Neurosurg. 2019. doi: 10.1016/j.wneu.2018.12.114.

7. World Health Organization (WHO). The World Health Report 2000 (Health Systems: Improving Performance). Geneva: WHO; 2000.

8. Al-Kalbani H, Al-Saadi T, Al-Kumzari A, Al-Bahrani H. Public's perception and satisfaction on the health care system in sultanate of Oman: a cross-sectional study. Ann Natl Acad Med Sci (India). 2020;56(04):214-9. doi: 10.1055/s-0040-1721554.

9. Mishra GP, Al Saadi T, Salhotra N, Biniwale S, Hadad $\mathrm{M}$, Hashim $\mathrm{M}$, et al. Brain and spinal tumors incidence annual audit 2017 of Dept of Neurosurgery Khoula Hospital Muscat Oman: a review. Am J Med Case Rep. 2018;6(7):128-31. doi: 10.12691/ajmcr-6-7-2.

10. Al-Saadi T, Al Sharqi A, Al Sharqi J, Al Blushi K, Al Rasbi $\mathrm{A}, \mathrm{Al}$-Farsi $\mathrm{M}$, et al. Leaving against medical advice among patients with brain tumours in the Middle East: Khoula hospital experience. Int Clin Neurosci J. 2020;7(4):179-84.

11. The Statistical Centre for the Cooperation Council for the Arab Countries of the Gulf. Available from: https://gccstat. org/en/.

12. Virta JJ, Satopää J, Luostarinen T, Raj R. One-year outcome after aneurysmal subarachnoid hemorrhage in elderly patients. World Neurosurg. 2020;143:e334-e43. doi: 10.1016/j.wneu.2020.07.127.

13. Shih RD, Ouslander JG. Intracranial hemorrhage in older adults: implications for fall risk assessment and prevention. J Am Geriatr Soc. 2020;68(5):953-5. doi: 10.1111/jgs.16399.

14. Cho DY, Chen CC, Lee HC, Lee WY, Lin HL. Glasgow Coma Scale and hematoma volume as criteria for treatment of putaminal and thalamic intracerebral hemorrhage. Surg Neurol. 2008;70(6):628-33. doi: 10.1016/j. surneu.2007.08.006. 